\title{
TORIC IDEALS OF SERIES AND PARALLEL CONNECTIONS OF MATROIDS
}

\author{
KAZUKI SHIBATA
}

\begin{abstract}
In 1980, White conjectured that the toric ideal associated to a matroid is generated by binomials corresponding to a symmetric exchange. In this paper, we prove that classes of matroids for which the toric ideal is generated by quadrics and that has quadratic Gröbner bases, are closed under series and parallel extensions, series and parallel connections, and 2-sums.
\end{abstract}

\section{INTRODUCTION}

A matroid has multiple equivalent definitions. We define a matroid as a collection of subsets that satisfies the exchange axiom: A matroid $M$ is a pair $(E, \mathcal{B})$, where $E=[d]=\{1, \ldots, d\}$ and $\mathcal{B}$ is a non-empty collection of subsets of $E$, which satisfies

- for every $B$ and $B^{\prime}$ in $\mathcal{B}$, for any $x \in B \backslash B^{\prime}$, there exists $y \in B^{\prime} \backslash B$ such that $(B \cup\{y\}) \backslash\{x\}$ is a member of $\mathcal{B}$.

The exchange axiom is equivalent to the following stronger axiom known as the symmetric exchange property.

- For every $B$ and $B^{\prime}$ in $\mathcal{B}$, for any $x \in B \backslash B^{\prime}$, there exists $y \in B^{\prime} \backslash B$ such that $(B \cup\{y\}) \backslash\{x\}$ and $\left(B^{\prime} \cup\{x\}\right) \backslash\{y\}$ are members of $\mathcal{B}$.

For a detailed introduction to matroid theory, see [12]. We call a member of $\mathcal{B}$ a basis of $M$. All the members of $\mathcal{B}$ have the same cardinality. This cardinality is said to be the rank of $M$ and is denoted by $\operatorname{rk}(M)$. Let $\mathcal{B}(M)=\left\{B_{1}, \ldots, B_{n}\right\}$ be the collection of bases of a matroid $M$ on $E$ and $\mathcal{D}_{M}=\left(\mathbf{b}_{1}, \ldots, \mathbf{b}_{n}\right)$ be an integer matrix satisfying $\mathbf{b}_{j}=\sum_{l \in B_{j}} \mathbf{e}_{l}$, where $\mathbf{e}_{l}$ is the $l$-th standard vector. Let $K$ be a field, and let $K[X]=K\left[x_{1}, \ldots, x_{n}\right]$ be the polynomial ring over $K$. We consider the ring homomorphism

$$
\pi_{M}: K[X] \rightarrow K[S]=K\left[s_{1}, \ldots, s_{d}\right], \quad x_{j} \mapsto S^{\mathbf{b}_{j}},
$$

where $S^{\mathbf{b}_{j}}=s_{1}^{b_{1}} \cdots s_{d}^{b_{d}}$ for $\mathbf{b}_{j}=\left(b_{1}, \ldots, b_{d}\right)$. The toric ideal $J_{\mathcal{D}_{M}}$, or briefly, $J_{M}$ is the kernel of $\pi_{M}$ (see [15]). The semigroup ring $R_{M}=K[X] / J_{M}$ is called the bases monomial ring of $M$, and it was introduced by $\mathrm{N}$. White. White proved that the bases monomial ring $R_{M}$ is normal and, in particular, it is a Cohen-Macaulay ring for any matroid $M$ (see [17]). White presented the following conjecture.

Conjecture $1.1([18])$. For any matroid $M, J_{M}$ is generated by the binomials $x_{i} x_{j}-x_{k} x_{l}$ such that the pair of bases $B_{k}, B_{l}$ can be obtained from the pair of $B_{i}$, $B_{j}$ by a symmetric exchange.

It is natural to ask for the following variant of Conjecture 1.1. 
Conjecture 1.2. For any matroid $M, J_{M}$ has a Gröbner basis consisting of the binomials $x_{i} x_{j}-x_{k} x_{l}$ such that the pair of bases $B_{k}, B_{l}$ can be obtained from the pair of $B_{i}, B_{j}$ by a symmetric exchange.

Conjecture 1.1 is true for graphic matroids [1], matroids with rank $\leq 3$ [7, sparse paving matroids [3] and strongly base orderable matroids [8]. Conjecture 1.2 is true for uniform matroids [14], matroids with rank $\leq 2$ [2, 11], graphic matroids without $M\left(K_{4}\right)$-minor [2] and lattice path matroids [13].

Let $\mathcal{M}_{\mathcal{Q G}}$ be the class of matroids for which the toric ideal $J_{M}$ has a quadratic Gröbner basis, let $\mathcal{M}_{\mathcal{Q}}$ be the class of matroids for which $J_{M}$ is generated by quadrics, and let $\mathcal{M}$ be the class of all matroids. The following is weaker than Conjecture 1.1 and Conjecture 1.2. However it is still open.

Conjecture 1.3. The equalities $\mathcal{M}_{\mathcal{Q G}}=\mathcal{M}_{\mathcal{Q}}=\mathcal{M}$ hold.

Conca proved that the class of transversal polymatroids is contained in $\mathcal{M}_{\mathcal{Q}}$ [5].

Let $M$ be a matroid on $E$, and let $\mathcal{B}(M)$ be the collection of bases of $M$. An element $i \in E$ is called a loop of $M$ if it does not belong to any basis of $M$. Dually, an element $i \in E$ is said to be a coloop of $M$ if it is contained in all the bases of $M$. Let $\mathcal{B}^{*}(M)=\{E \backslash B \mid B \in \mathcal{B}(M)\}$. Then a pair $\left(E, \mathcal{B}^{*}(M)\right)$ is a matroid, and it is called the dual of $M$ and is denoted as $M^{*}$.

Let $M$ and $\mathcal{B}(M)$ be as above, and let $c \in E$. We consider the following collection of subsets of $E \backslash\{c\}$ :

$$
\mathcal{B}(M) \backslash c= \begin{cases}\{B \backslash\{c\} \mid B \in \mathcal{B}(M)\} & \text { if } c \text { is a coloop of } M, \\ \{B \mid c \notin B \in \mathcal{B}(M)\} & \text { otherwise. }\end{cases}
$$

A pair $(E \backslash\{c\}, \mathcal{B}(M) \backslash c)$ is a matroid, and it is called the deletion of $c$ from $M$ and is denoted as $M \backslash c$. Dually, let $M / c$, the contraction of $c$ from $M$, be given by $M / c=\left(M^{*} \backslash c\right)^{*}$. We call a matroid $M^{\prime}$ a minor of a matroid $M$ if $M^{\prime}$ can be obtained from $M$ by a finite sequence of contractions and deletions.

Let $M_{1}$ and $M_{2}$ be matroids with $E_{1} \cap E_{2}=\emptyset$. Let $\mathcal{B}\left(M_{1}\right)$ and $\mathcal{B}\left(M_{2}\right)$ be collections of bases of $M_{1}$ and $M_{2}$, and let

$$
\mathcal{B}\left(M_{1}\right) \oplus \mathcal{B}\left(M_{2}\right)=\left\{B \cup D \mid B \in \mathcal{B}\left(M_{1}\right), D \in \mathcal{B}\left(M_{2}\right)\right\} .
$$

Then a pair $\left(E, \mathcal{B}\left(M_{1}\right) \oplus \mathcal{B}\left(M_{2}\right)\right)$, where $E=E_{1} \cup E_{2}$, is a matroid. This matroid is called the direct sum of $M_{1}$ and $M_{2}$, and it is denoted as $M_{1} \oplus M_{2}$.

Proposition 1.4 ([2, 18]). Classes $\mathcal{M}_{\mathcal{Q G}}$ and $\mathcal{M}_{\mathcal{Q}}$ are closed under the duality, taking minors and direct sums.

The outline of this paper is as follows. In Section 2, we describe how to compute generating sets and Gröbner bases for the toric ideal of series and parallel extensions. In Section 3, we use the results from Section 2 to form generating sets and Gröbner bases for the toric ideal of series and parallel connections and 2-sums. 


\section{A SERIES AND PARALLEL EXTENSION OF A MATROID}

Let $M$ be a matroid on $E=[d]$, and let $\mathcal{B}(M)$ be the collection of bases of $M$. Then a series extension of $M$ at $c \in E$ by $d+1$ is a matroid on $E \cup\{d+1\}$ which has

$$
\{B \cup\{d+1\} \mid B \in \mathcal{B}(M)\} \cup\{B \cup\{c\} \mid c \notin B \in \mathcal{B}(M)\}
$$

as the collection of bases and is denoted as $M+{ }_{c}(d+1)$. Dually, we call a matroid $\left(M^{*}+{ }_{c}(d+1)\right)^{*}$ a parallel extension of $M$ at $c$ by $d+1$. A series-parallel extension of $M$ is any matroid derived from $M$ by a finite sequence of series and parallel extensions. We suppose that $M$ does not have $c \in E$ as a coloop. Let $\mathcal{B}(M)=$ $\left\{B_{1}, \ldots, B_{\gamma}, \ldots, B_{n}\right\}$ be the collection of bases of $M$, where $c \notin B_{j}$ for $j \in[\gamma]$ and $c \in B_{j}$ for $j \in[n] \backslash[\gamma]$. We renumber the bases of $M$, if necessary. Let $\mathcal{D}_{M}=\left(\mathbf{b}_{1}, \ldots, \mathbf{b}_{n}\right)$ denote an integer matrix arising from $M$. Now we consider a new integer matrix

$$
\widetilde{\mathcal{D}}_{M}=\left(\begin{array}{ccc|ccc}
\mathbf{b}_{1} & \cdots & \mathbf{b}_{n} & \mathbf{b}_{1} & \cdots & \mathbf{b}_{\gamma} \\
\mathbf{e}_{1} & \cdots & \mathbf{e}_{1} & \mathbf{e}_{2} & \cdots & \mathbf{e}_{2}
\end{array}\right) .
$$

We define the ring homomorphism $\widetilde{\pi}_{M}$ as follows:

$$
\begin{aligned}
\tilde{\pi}_{M}: K[X]=K\left[x_{1}^{1}, \ldots, x_{n}^{1}, x_{1}^{2}, \ldots, x_{\gamma}^{2}\right] & \rightarrow K\left[S, w_{1}, w_{2}\right], \\
x_{j}^{i} & \mapsto S^{\mathbf{b}_{j}} w_{i} .
\end{aligned}
$$

Let $\omega \in \mathbb{Z}_{\geq 0}^{n}$, and let $\prec$ be an arbitrary monomial order. We define a new monomial order $\prec_{\omega}$ as follows:

$$
X^{\mathbf{a}} \prec_{\omega} X^{\mathbf{b}} \Leftrightarrow\left\{\begin{array}{l}
\omega \cdot \mathbf{a}<\omega \cdot \mathbf{b} \text {; or } \\
\omega \cdot \mathbf{a}=\omega \cdot \mathbf{b} \text { and } X^{\mathbf{a}} \prec X^{\mathbf{b}},
\end{array}\right.
$$

for $\mathbf{a}, \mathbf{b} \in \mathbb{Z}_{\geq 0}^{n}$. We call a monomial order $\prec_{\omega}$ a weight order on $K\left[x_{1}, \ldots, x_{n}\right]$. We use the following useful result:

Proposition 2.1 ([14, Proposition 1.11]). For any monomial order $\prec$ and any ideal $I \subset K\left[x_{1}, \ldots, x_{n}\right]$, there exists a vector $w \in \mathbb{Z}_{\geq 0}^{n}$ such that $\operatorname{in}_{\omega}(I)=\operatorname{in}_{\prec}(I)$.

Let $\mathbf{F}$ be a homogeneous generating set for $J_{\mathcal{D}_{M}}$, and let

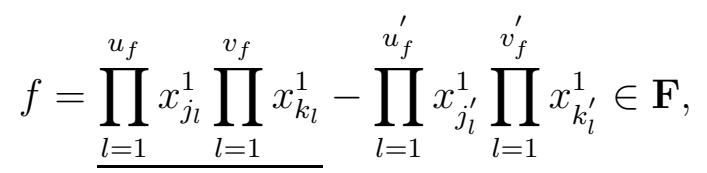

where $j_{l}, j_{l}^{\prime} \in[\gamma], k_{l}, k_{l}^{\prime} \in[n] \backslash[\gamma]$. However, if $v_{f} \neq v_{f}^{\prime}$, then $\pi_{M}(f) \neq 0$ since the $c$-th entry of $\sum_{l=1}^{v_{f}} \mathbf{b}_{k_{l}}$ does not coincide with the $c$-th entry of $\sum_{l=1}^{v_{f}^{\prime}} \mathbf{b}_{k_{l}^{\prime}}$, and the c-th entries of $\sum_{l=1}^{u_{f}} \mathbf{b}_{j_{l}}$ and $\sum_{l=1}^{u_{f}^{\prime}} \mathbf{b}_{j_{l}^{\prime}}$ are zero. Therefore $u_{f}=u_{f}^{\prime}$ and $v_{f}=v_{f}^{\prime}$. Now let $I=\left(i_{1}, \ldots, i_{u_{f}}\right) \in\{1,2\}^{u_{f}}$ and consider the binomial $f^{I} \in K[X]$ defined by

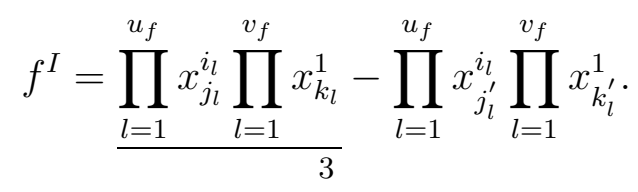


Since $f \in J_{\mathcal{D}_{M}}$, the new homogeneous binomial $f^{I} \in J_{\widetilde{\mathcal{D}}_{M}}$. We set

$$
\left.\widetilde{\mathbf{F}}=\left\{f^{I} \mid f \in \mathbf{F}, I \in\{1,2\}^{u_{f}}\right\} \cup \underline{\left\{x_{j_{2}}^{1} x_{j_{1}}^{2}\right.}-x_{j_{1}}^{1} x_{j_{2}}^{2} \mid 1 \leq j_{1}<j_{2} \leq \gamma\right\} .
$$

Lemma 2.2. Let $M$ be a matroid on $E$, and let $\mathbf{F}$ be a Gröbner basis for $J_{\mathcal{D}_{M}}$. Then $\widetilde{\mathbf{F}}$ is a Gröbner basis for $J_{\widetilde{\mathcal{D}}_{M}}$.

Proof. First, it is easy to see that $\widetilde{\mathbf{F}} \subset J_{\widetilde{\mathcal{D}}_{M}}$. Let $\omega=\left(\omega_{1}^{1}, \ldots, \omega_{n}^{1}\right)$ be a weight vector. We denote the underlined monomial of $f$ as the initial monomial of $f$ with respect to a weight order induced by $\omega$. Let $\widetilde{\omega}=\left(\omega_{1}^{1}, \ldots, \omega_{n}^{1}, \omega_{1}^{2}, \ldots, \omega_{\gamma}^{2}\right)$ denote a weight vector satisfying $\omega_{j}^{1}=\omega_{j}^{2}$ for $j \in[\gamma]$. Then the underlined monomial of $f^{I}$ is the initial monomial of $f^{I}$ with respect to a weight order $\prec_{\widetilde{\omega}}$. We choose a tie-breaking monomial order on $K[X]$ that makes the monomial $x_{j_{2}}^{1} x_{j_{1}}^{2}$ for $1 \leq j_{1}<j_{2} \leq \gamma$ the initial monomial. Let $\operatorname{in}(\mathbf{F})=\left\langle\operatorname{in}_{\omega}(f) \mid f \in \mathbf{F}\right\rangle$ and $\operatorname{in}(\widetilde{\mathbf{F}})=\left\langle\operatorname{in}_{\prec \widetilde{\omega}}(f) \mid f \in \widetilde{\mathbf{F}}\right\rangle$. Let $u$ and $v$ be monomials which are not in $\operatorname{in}(\widetilde{\mathbf{F}})$ :

$$
\begin{aligned}
& u=\prod_{l=1}^{m_{1}}\left(x_{i_{l}}^{1}\right)^{p_{l}} \prod_{l=1}^{m_{2}}\left(x_{j_{l}}^{2}\right)^{q_{l}} \prod_{l=1}^{m_{3}}\left(x_{k_{l}}^{1}\right)^{r_{l}} \\
& v=\prod_{l=1}^{m_{1}^{\prime}}\left(x_{i_{l}^{\prime}}^{1}\right)^{p_{l}^{\prime}} \prod_{l=1}^{m_{2}^{\prime}}\left(x_{j_{l}^{\prime}}^{2}\right)^{q_{l}^{\prime}} \prod_{l=1}^{m_{3}^{\prime}}\left(x_{k_{l}^{\prime}}^{1}\right)^{r_{l}^{\prime}}
\end{aligned}
$$

where $p_{l}, q_{l}, r_{l}, p_{l}^{\prime}, q_{l}^{\prime}, r_{l}^{\prime} \in \mathbb{Z}_{>0}$ for any $l$, and $\mathcal{I}=\left\{i_{1}, \ldots, i_{m_{1}}\right\}, \mathcal{I}^{\prime}=\left\{i_{1}^{\prime}, \ldots, i_{m_{1}^{\prime}}^{\prime}\right\}$, $\mathcal{J}=\left\{j_{1}, \ldots, j_{m_{2}}\right\}$, and $\mathcal{J}^{\prime}=\left\{j_{1}^{\prime}, \ldots, j_{m_{2}^{\prime}}^{\prime}\right\}$ are subsets of $[\gamma]$ with cardinalities $m_{1}$, $m_{1}^{\prime}, m_{2}$, and $m_{2}^{\prime}$, respectively; and $\mathcal{K}=\left\{k_{1}, \ldots, k_{m_{3}}\right\}$ and $\mathcal{K}^{\prime}=\left\{k_{1}^{\prime}, \ldots, k_{m_{3}^{\prime}}^{\prime}\right\}$ are subsets of $[n] \backslash[\gamma]$ with cardinalities $m_{3}$ and $m_{3}^{\prime}$, respectively. Since neither $u$ nor $v$ is divided by $x_{j_{2}}^{1} x_{j_{1}}^{2}$ for $1 \leq j_{1}<j_{2} \leq \gamma$, it follows that $i_{l} \leq j_{l^{\prime}}$ for $l \in\left[m_{1}\right]$ and $l^{\prime} \in\left[m_{2}\right]$, and $i_{l}^{\prime} \leq j_{l^{\prime}}^{\prime}$ for $l \in\left[m_{1}^{\prime}\right]$ and $l^{\prime} \in\left[m_{2}^{\prime}\right]$. We suppose that $\widetilde{\pi}_{M}(u)=\widetilde{\pi}_{M}(v)$, i.e., $p+q=p^{\prime}+q^{\prime}, r=r^{\prime}$ and

$$
\sum_{l=1}^{m_{1}} p_{l} \mathbf{b}_{i_{l}}+\sum_{l=1}^{m_{2}} q_{l} \mathbf{b}_{j_{l}}+\sum_{l=1}^{m_{3}} r_{l} \mathbf{b}_{k_{l}}=\sum_{l=1}^{m_{1}^{\prime}} p_{l}^{\prime} \mathbf{b}_{i_{l}^{\prime}}+\sum_{l=1}^{m_{2}^{\prime}} q_{l}^{\prime} \mathbf{b}_{j_{l}^{\prime}}+\sum_{l=1}^{m_{3}^{\prime}} r_{l}^{\prime} \mathbf{b}_{k_{l}^{\prime}}
$$

Here we set $p=\sum_{l=1}^{m_{1}} p_{l}, q=\sum_{l=1}^{m_{2}} q_{l}, r=\sum_{l=1}^{m_{3}} r_{l}, p^{\prime}=\sum_{l=1}^{m_{1}^{\prime}} p_{l}^{\prime}, q^{\prime}=\sum_{l=1}^{m_{2}^{\prime}} q_{l}^{\prime}$, and $r^{\prime}=\sum_{l=1}^{m_{3}^{\prime}} r_{l}^{\prime}$. Therefore it follows that $\pi_{M}\left(u^{\prime}\right)=\pi_{M}\left(v^{\prime}\right)$, where

$$
\begin{aligned}
& u^{\prime}=\prod_{l=1}^{m_{1}}\left(x_{i_{l}}^{1}\right)^{p_{l}} \prod_{l=1}^{m_{2}}\left(x_{j_{l}}^{1}\right)^{q_{l}} \prod_{l=1}^{m_{3}}\left(x_{k_{l}}^{1}\right)^{r_{l}}, \\
& v^{\prime}=\prod_{l=1}^{m_{1}^{\prime}}\left(x_{i_{l}^{\prime}}^{1}\right)^{p_{l}^{\prime}} \prod_{l=1}^{m_{2}^{\prime}}\left(x_{j_{l}^{\prime}}^{1}\right)^{q_{l}^{\prime}} \prod_{l=1}^{m_{3}^{\prime}}\left(x_{k_{l}^{\prime}}^{1}\right)^{r_{l}^{\prime}} .
\end{aligned}
$$

Hence $u^{\prime}-v^{\prime}$ belongs to $J_{\mathcal{D}_{M}}$. If $u^{\prime}$ or $v^{\prime}$ belongs to in $(\mathbf{F})$, then $u^{\prime}$ or $v^{\prime}$ is in $\operatorname{in}(\widetilde{\mathbf{F}})$. In particular, $u$ or $v$ belongs to $\operatorname{in}(\widetilde{\mathbf{F}})$. This is a contradiction. Therefore neither $u^{\prime}$ 
nor $v^{\prime}$ belongs to $\operatorname{in}(\mathbf{F})$. Since $\mathbf{F}$ is a Gröbner basis for $J_{\mathcal{D}_{M}}$, it follows that $u^{\prime}=v^{\prime}$. In particular, $\mathcal{I}=\mathcal{I}^{\prime}, \mathcal{J}=\mathcal{J}^{\prime}, \mathcal{K}=\mathcal{K}^{\prime}, p_{l}=p_{l}^{\prime}, q_{l}=q_{l}^{\prime}$, and $r_{l}=r_{l}^{\prime}$ for any $l$. Thus $u=v$. Therefore $\widetilde{\mathbf{F}}$ is a Gröbner basis for $J_{\widetilde{\mathcal{D}}_{M}}$.

Lemma 2.3. Let $M$ be a matroid on $E$. If $\mathbf{F}$ is a homogeneous generating set for $J_{\mathcal{D}_{M}}$, then $\widetilde{\mathbf{F}}$ is a generating set for $J_{\widetilde{\mathcal{D}}_{M}}$.

Proof. We assume that $\mathbf{F}$ and $\mathbf{F}^{\prime}$ are generating sets for $J_{\mathcal{D}_{M}}$. Then $\widetilde{\mathbf{F}}$ and $\widetilde{\mathbf{F}}^{\prime}$ generate the same ideal. In particular, this holds if $\mathbf{F}^{\prime}$ is a Gröbner basis for $J_{\mathcal{D}_{M}}$. Thus $\langle\widetilde{\mathbf{F}}\rangle=\left\langle\widetilde{\mathbf{F}}^{\prime}\right\rangle$. By Lemma 2.2, if $\mathbf{F}^{\prime}$ is a Gröbner basis for $J_{\mathcal{D}_{M}}$, then $\widetilde{\mathbf{F}}^{\prime}$ is a generating set for $J_{\widetilde{\mathcal{D}}_{M}}$, since $\widetilde{\mathbf{F}}^{\prime}$ is a Gröbner basis for $J_{\widetilde{\mathcal{D}}_{M}}$.

Theorem 2.4. Let $M$ be a matroid on $E$, and let $M+{ }_{c}(d+1)$ denote a series extension of $M$ at $c$ by $d+1$. Then $\widetilde{\mathbf{F}}$ becomes a generating set (resp. a Gröbner basis) for $J_{M+c}(d+1)$.

Proof. By elementary row operations on $\widetilde{\mathcal{D}}_{M}$, we obtain the integer matrix arising from $M+{ }_{c}(d+1)$.

Remark 2.5. If $c$ is a coloop of $M$, then $J_{M+c(d+1)}=J_{M}$.

Theorem 2.6. Classes $\mathcal{M}_{\mathcal{Q G}}$ and $\mathcal{M}_{\mathcal{Q}}$ are closed under series and parallel extensions.

\section{A SERIES AND PARALLEL CONNECTION OF MATROIDS}

Let $M_{1}$ and $M_{2}$ be matroids with $E_{1} \cap E_{2}=\{c\}$ and $E=E_{1} \cup E_{2}$. Suppose that for both $M_{1}$ and $M_{2}, c$ is neither a loop nor a coloop. Let

$$
\begin{aligned}
\mathcal{B}_{\mathcal{S}}= & \left\{B \cup D \mid B \in \mathcal{B}\left(M_{1}\right), D \in \mathcal{B}\left(M_{2}\right), B \cap D=\emptyset\right\}, \\
\mathcal{B}_{\mathcal{P}}= & \left\{B \cup D \mid B \in \mathcal{B}\left(M_{1}\right), D \in \mathcal{B}\left(M_{2}\right), c \in B \cap D\right\} \\
& \cup\left\{(B \cup D) \backslash\{c\} \mid B \in \mathcal{B}\left(M_{1}\right), D \in \mathcal{B}\left(M_{2}\right), c \text { is in exactly one of } B \text { and } D\right\} .
\end{aligned}
$$

Then pairs $\left(E, \mathcal{B}_{\mathcal{S}}\right)$ and $\left(E, \mathcal{B}_{\mathcal{P}}\right)$ are matroids. These matroids are said to be the series and parallel connections of $M_{1}$ and $M_{2}$ with respect to the basepoint $c$. We denote them as $S\left(\left(M_{1} ; c\right),\left(M_{2} ; c\right)\right)$ and $P\left(\left(M_{1} ; c\right),\left(M_{2} ; c\right)\right)$, or briefly, $S\left(M_{1}, M_{2}\right)$ and $P\left(M_{1}, M_{2}\right)$ [12, Proposition 7.1.13].

On the other hand, when $c$ is a loop of $M_{1}$, then we define

$$
P\left(M_{1}, M_{2}\right)=M_{1} \oplus\left(M_{2} / c\right) \quad \text { and } \quad S\left(M_{1}, M_{2}\right)=\left(M_{1} / c\right) \oplus M_{2} .
$$

When $c$ is a coloop of $M_{1}$, then we define

$$
P\left(M_{1}, M_{2}\right)=\left(M_{1} \backslash c\right) \oplus M_{2} \quad \text { and } \quad S\left(M_{1}, M_{2}\right)=M_{1} \oplus\left(M_{2} \backslash c\right)
$$

(see [12, 7.1.5 - 7.1.8]). Moreover, the 2-sum $M_{1} \oplus_{2} M_{2}$ of $M_{1}$ and $M_{2}$ is $S\left(M_{1}, M_{2}\right) / c$, or equivalently, $P\left(M_{1}, M_{2}\right) \backslash c$, where $c$ is neither a loop nor a coloop of either $M_{1}$ or $M_{2}$. 
Let $M_{1}$ and $M_{2}$ be matroids on $E_{1}=\left[d_{1}\right]$ and $E_{2}=\left[d_{2}\right]$. We identify the set $\left[d_{2}\right]$ with the set $\left\{d_{1}+1, \ldots, d_{1}+d_{2}\right\}$. Assume that $c_{i} \in E_{i}$ is not a coloop of $M_{i}$ for $i \in[2]$. Let

$$
\mathcal{B}\left(M_{1}\right)=\left\{B_{1}, \ldots, B_{\gamma_{1}}, \ldots, B_{n_{1}}\right\} \quad \text { and } \quad \mathcal{B}\left(M_{2}\right)=\left\{D_{1}, \ldots, D_{\gamma_{2}}, \ldots, D_{n_{2}}\right\}
$$

be collections of bases of $M_{1}$ and $M_{2}$, where $c_{1} \notin B_{j}$ for $j \in\left[\gamma_{1}\right]$ and $c_{2} \notin D_{k}$ for $k \in\left[\gamma_{2}\right]$. Let $\mathcal{D}_{M_{1}}=\left(\mathbf{b}_{1}, \ldots, \mathbf{b}_{n_{1}}\right)$ and $\mathcal{D}_{M_{2}}=\left(\mathbf{d}_{1}, \ldots, \mathbf{d}_{n_{2}}\right)$ be two integer matrices arising from $M_{1}$ and $M_{2}$. We define ring homomorphisms $\pi_{M_{1}}$ and $\pi_{M_{2}}$ by setting

$$
\begin{array}{rlrl}
\pi_{M_{1}}: K\left[x_{1}^{1}, \ldots, x_{n_{1}}^{1}\right] & \rightarrow K[S] & x_{j}^{1} \mapsto S^{\mathbf{b}_{j}} \\
\pi_{M_{2}}: K\left[y_{1}^{2}, \ldots, y_{n_{2}}^{2}\right] & \rightarrow K[T] & y_{k}^{2} & \mapsto T^{\mathbf{d}_{k}} .
\end{array}
$$

Similar to what we did in Section 2, we consider two integer matrices

$$
\begin{aligned}
& \widetilde{\mathcal{D}}_{M_{1}}=\left(\begin{array}{ccc|ccc}
\mathbf{b}_{1} & \cdots & \mathbf{b}_{n_{1}} & \mathbf{b}_{1} & \cdots & \mathbf{b}_{\gamma_{1}} \\
\mathbf{e}_{1} & \cdots & \mathbf{e}_{1} & \mathbf{e}_{2} & \cdots & \mathbf{e}_{2}
\end{array}\right), \\
& \widetilde{\mathcal{D}}_{M_{2}}=\left(\begin{array}{ccc|ccc}
\mathbf{d}_{1} & \cdots & \mathbf{d}_{\gamma_{2}} & \mathbf{d}_{1} & \cdots & \mathbf{d}_{n_{2}} \\
\mathbf{e}_{1} & \cdots & \mathbf{e}_{1} & \mathbf{e}_{2} & \cdots & \mathbf{e}_{2}
\end{array}\right) .
\end{aligned}
$$

We define ring homomorphisms $\widetilde{\pi}_{M_{1}}$ and $\widetilde{\pi}_{M_{2}}$ as follows:

$$
\begin{aligned}
\widetilde{\pi}_{M_{1}}: K[X]=K\left[x_{1}^{1}, \ldots, x_{n_{1}}^{1}, x_{1}^{2}, \ldots, x_{\gamma_{1}}^{2}\right] & \rightarrow K\left[S, w_{1}, w_{2}\right], & x_{j}^{i} \mapsto S^{\mathbf{b}_{j}} w_{i}, \\
\widetilde{\pi}_{M_{2}}: K[Y]=K\left[y_{1}^{1}, \ldots, y_{\gamma_{2}}^{1}, y_{1}^{2}, \ldots, y_{n_{2}}^{2}\right] & \rightarrow K\left[T, w_{1}, w_{2}\right], & y_{k}^{i} \mapsto T^{\mathbf{d}_{k}} w_{i} .
\end{aligned}
$$

Moreover, consider the integer matrix

$$
\widetilde{\mathcal{D}}=\left(\begin{array}{ccc}
\mathbf{b}_{1} & & \mathbf{b}_{1} \\
\mathbf{d}_{1} & \ldots & \mathbf{d}_{\gamma_{2}} \\
\mathbf{e}_{1} & & \mathbf{e}_{1}
\end{array}|\ldots| \begin{array}{ccc|ccc}
\mathbf{b}_{n_{1}} & \ldots & \mathbf{b}_{n_{1}} & \mathbf{b}_{1} & & \mathbf{b}_{1} \\
\mathbf{d}_{1} & \ldots & \mathbf{d}_{\gamma_{2}} & \mathbf{d}_{1} & \ldots & \mathbf{d}_{n_{2}} \\
\mathbf{e}_{1} & & \mathbf{e}_{1} & \mathbf{e}_{2} & & \mathbf{e}_{2}
\end{array}|\ldots| \begin{array}{ccc}
\mathbf{b}_{\gamma_{1}} & & \mathbf{b}_{\gamma_{1}} \\
\mathbf{d}_{1} & \ldots & \mathbf{d}_{n_{2}} \\
\mathbf{e}_{2} & & \mathbf{e}_{2}
\end{array}\right) .
$$

Let $K[Z]=K\left[z_{j k}^{i} \mid i=1,2, j \in\left[\alpha_{i}\right], k \in\left[\beta_{i}\right]\right]$ be the polynomial ring over $K$, where $\left(\alpha_{1}, \alpha_{2}\right)=\left(n_{1}, \gamma_{1}\right)$ and $\left(\beta_{1}, \beta_{2}\right)=\left(\gamma_{2}, n_{2}\right)$. The ring homomorphism $\widetilde{\pi}$ is defined by

$$
\tilde{\pi}: K[Z] \rightarrow K\left[S, T, w_{1}, w_{2}\right], \quad z_{j k}^{i} \mapsto S^{\mathbf{b}_{j}} T^{\mathbf{d}_{k}} w_{i} .
$$

Let $\mathbf{F}_{1}$ and $\mathbf{F}_{2}$ be homogeneous generating sets for $J_{\mathcal{D}_{M_{1}}}$ and $J_{\mathcal{D}_{M_{2}}}$, respectively. Then we define $\widetilde{\mathbf{F}}_{1}$ and $\widetilde{\mathbf{F}}_{2}$ in a way analogous to what we did in Section 2 , Let

$$
f=\prod_{l=1}^{u_{f}} x_{j_{l}^{1}}^{i_{l}}-\prod_{l=1}^{u_{f}} x_{j_{l}^{2}}^{i_{l}} \in \widetilde{\mathbf{F}}_{1}
$$

and let $k=\left(k_{1}, \ldots, k_{u_{f}}\right)$, with $k_{l} \in\left[\beta_{i_{l}}\right]$ for $1 \leq l \leq u_{f}$. We consider the binomial $f_{k} \in K[Z]$ defined by

$$
f_{k}=\prod_{l=1}^{u_{f}} z_{j_{l}^{1} k_{l}}^{i_{l}}-\prod_{l=1}^{u_{f}} z_{j_{l}^{2} k_{l}}^{i_{l}}
$$


Since $f \in J_{\widetilde{\mathcal{D}}_{M_{1}}}$, the new homogeneous binomial $f_{k} \in J_{\widetilde{\mathcal{D}}}$. If $\widetilde{\mathbf{F}}_{1}$ is any set of binomials in $J_{\widetilde{\mathcal{D}}_{M_{1}}}$, then

$$
\operatorname{Lift}\left(\widetilde{\mathbf{F}}_{1}\right)=\left\{f_{k} \mid f \in \widetilde{\mathbf{F}}_{1}, k \in \prod_{l=1}^{u_{f}}\left[\beta_{i_{l}}\right]\right\} .
$$

We define $\operatorname{Lift}\left(\widetilde{\mathbf{F}}_{2}\right)$ in an analogous way. Furthermore, the quadratic binomial set $\operatorname{Quad}\left(\widetilde{\mathcal{D}}_{M_{1}}, \widetilde{\mathcal{D}}_{M_{2}}\right)$ is defined by

$$
\operatorname{Quad}\left(\widetilde{\mathcal{D}}_{M_{1}}, \widetilde{\mathcal{D}}_{M_{2}}\right)=\left\{z_{j_{1} k_{2}}^{i} z_{j_{2} k_{1}}^{i}-z_{j_{1} k_{1}}^{i} z_{j_{2} k_{2}}^{i} \mid i=1,2, \begin{array}{l}
1 \leq j_{1}<j_{2} \leq \alpha_{i} \\
1 \leq k_{1}<k_{2} \leq \beta_{i}
\end{array}\right\}
$$

We set $\widetilde{N}=\operatorname{Lift}\left(\widetilde{\mathbf{F}}_{1}\right) \cup \operatorname{Lift}\left(\widetilde{\mathbf{F}}_{2}\right) \cup \operatorname{Quad}\left(\widetilde{\mathcal{D}}_{M_{1}}, \widetilde{\mathcal{D}}_{M_{2}}\right)$.

Lemma 3.1. Let $M_{1}$ and $M_{2}$ be matroids on $E_{1}=\left[d_{1}\right]$ and $E_{2}=\left[d_{2}\right]$, respectively; and assume that $c_{i} \in E_{i}$ is not a coloop of $M_{i}$ for $i=1,2$. Let $S\left(M_{1}, M_{2}\right)$ be a series connection of $M_{1}$ and $M_{2}$ with respect to the basepoint $c=c_{1}=c_{2}$. Then

$$
N=\widetilde{\mathrm{N}} \cap K[\widehat{Z}]
$$

is a generating set for $J_{S\left(M_{1}, M_{2}\right)}$. Here we set $K[\widehat{Z}]=K\left[z_{j k}^{i} \mid i=1,2, j \in\left[\alpha_{i}\right], k \in\right.$ $\left.V_{i}\right]$, where $V_{1}=\left[\gamma_{2}\right]$ and $V_{2}=\left[n_{2}\right] \backslash\left[\gamma_{2}\right]$. Moreover, if $\mathbf{F}_{1}$ and $\mathbf{F}_{2}$ are Gröbner bases for $J_{\mathcal{D}_{M_{1}}}$ and $J_{\mathcal{D}_{M_{2}}}$, then there exists a monomial order such that $\mathrm{N}$ is a Gröbner basis for $J_{S\left(M_{1}, M_{2}\right)}$.

For the proof of Lemma 3.1 , we use results in [6, 16].

Let $r>0$ be a positive integer, and let $\alpha, \beta \in \mathbb{Z}_{>0}^{r}$ be two vectors of positive integers. Let

$$
K[X]=K\left[x_{j}^{i} \mid i \in[r], j \in\left[\alpha_{i}\right]\right] \text { and } K[Y]=K\left[y_{k}^{i} \mid i \in[r], k \in\left[\beta_{i}\right]\right]
$$

be two multigraded polynomial rings with the multigrading $\operatorname{deg}\left(x_{j}^{i}\right)=\operatorname{deg}\left(y_{k}^{i}\right)=$ $\mathbf{a}^{i} \in \mathbb{Z}^{d}$, where $\alpha_{i}$ (resp. $\left.\beta_{i}\right)$ is the $i$-th entry of $\alpha$ (resp. $\beta$ ). We write $\mathcal{A}=$ $\left\{\mathbf{a}^{1}, \ldots, \mathbf{a}^{r}\right\}$ and assume that $\mathcal{A}$ is linearly independent. If $I$ and $J$ are homogeneous ideals of $K[X]$ and $K[Y]$, then the quotient rings $R_{1}=K[X] / I$ and $R_{2}=K[Y] / J$ are also multigraded by $\mathcal{A}$. Consider the polynomial ring

$$
K[Z]=K\left[z_{j k}^{i} \mid i \in[r], j \in\left[\alpha_{i}\right], k \in\left[\beta_{i}\right]\right]
$$

and consider the ring homomorphism

$$
\phi_{I, J}: K[Z] \rightarrow R_{1} \otimes_{K} R_{2}, \quad z_{j k}^{i} \mapsto x_{j}^{i} \otimes y_{k}^{i} .
$$

The kernel of $\phi_{I, J}$ is called the toric fiber product of $I$ and $J$. It is denoted as $I \times_{\mathcal{A}} J=\operatorname{ker}\left(\phi_{I, J}\right)$. The following result is in [16, Theorem 12 and Corollary 14].

Proposition 3.2. Suppose that the set $\mathcal{A}$ of degree vectors is linearly independent. Let $\mathbf{F}_{1}$ and $\mathbf{F}_{2}$ be homogeneous generating sets for $I$ and $J$, respectively. Then

$$
\mathrm{N}=\operatorname{Lift}\left(\mathbf{F}_{1}\right) \cup \operatorname{Lift}\left(\mathbf{F}_{2}\right) \cup \operatorname{Quad}_{\mathcal{A}}
$$

is a homogeneous generating set for $I \times_{\mathcal{A}} J$. Moreover, if $\mathbf{F}_{1}$ and $\mathbf{F}_{2}$ are Gröbner bases of $I$ and $J$, then there exists a monomial order such that $\mathrm{N}$ is a Gröbner basis for $I \times_{\mathcal{A}} J$. The sets $\operatorname{Lift}\left(\mathbf{F}_{1}\right)$, $\operatorname{Lift}\left(\mathbf{F}_{2}\right)$, and $\operatorname{Quad}_{\mathcal{A}}$ are defined in [16]. 
On the other hand, if $I$ and $J$ are toric ideals, then $I \times_{\mathcal{A}} J$ is also a toric ideal. If $K[S]$ and $K[T]$ are polynomial rings, and

$$
\begin{aligned}
& \phi: K[X] \rightarrow K[S], \quad x_{j}^{i} \mapsto f_{j}^{i}(S), \\
& \psi: K[Y] \rightarrow K[T], \quad y_{k}^{i} \mapsto g_{k}^{i}(T)
\end{aligned}
$$

are ring homomorphisms, then we can form the toric fiber product homomorphism

$$
\phi \times_{\mathcal{A}} \psi: K[Z] \rightarrow K[S, T], \quad z_{j k}^{i} \mapsto f_{j}^{i}(S) g_{k}^{i}(T) .
$$

If $I=\operatorname{ker}(\phi)$ and $J=\operatorname{ker}(\psi)$ and both ideals are homogeneous with respect to the grading by $\mathcal{A}$, then $I \times_{\mathcal{A}} J=\operatorname{ker}\left(\phi \times_{\mathcal{A}} \psi\right)$ (see [6]).

Proof of Lemma 3.1. Let $\mathbf{F}_{1}$ and $\mathbf{F}_{2}$ be generating sets (resp. Gröbner bases) for $J_{\mathcal{D}_{M_{1}}}$ and $J_{\mathcal{D}_{M_{2}}}$. From Lemma 2.2, Lemma 2.3, and Proposition 3.2, $\widetilde{\mathrm{N}}$ is a generating set (resp. a Gröbner basis) for $J_{\widetilde{\mathcal{D}}}$. Now we consider two integer matrices

$$
\begin{aligned}
& \widetilde{\mathcal{D}}^{\prime}=\left(\begin{array}{ccc}
\mathbf{b}_{1} & & \mathbf{b}_{1} \\
\mathbf{d}_{1} & \cdots & \mathbf{d}_{\gamma_{2}} \\
\mathbf{e}_{1} & & \mathbf{e}_{1}
\end{array}|\cdots| \begin{array}{ccc|ccc}
\mathbf{b}_{n_{1}} & & \mathbf{b}_{n_{1}} & \mathbf{b}_{1} & & \mathbf{b}_{1} \\
\mathbf{d}_{1} & \cdots & \mathbf{d}_{\gamma_{2}} & \mathbf{d}_{1} & \cdots & \mathbf{d}_{n_{2}} \\
\mathbf{e}_{1} & & \mathbf{e}_{1} & \mathbf{c}_{1} & & \mathbf{c}_{n_{2}}
\end{array}|\cdots| \begin{array}{cccc}
\mathbf{b}_{\gamma_{1}} & & \mathbf{b}_{\gamma_{1}} \\
\mathbf{d}_{1} & \cdots & \mathbf{d}_{n_{2}} \\
\mathbf{c}_{1} & & \mathbf{c}_{n_{2}}
\end{array}\right), \\
& \mathcal{D}=\left(\begin{array}{ccc}
\mathbf{b}_{1} & & \mathbf{b}_{1} \\
\mathbf{d}_{1} & \cdots & \mathbf{d}_{\gamma_{2}}
\end{array}|\cdots| \begin{array}{cccc|ccc|c|cc}
\mathbf{b}_{n_{1}} & & \mathbf{b}_{n_{1}} & \mathbf{b}_{1} & & \mathbf{b}_{1} \\
\mathbf{d}_{1} & \cdots & \mathbf{d}_{\gamma_{2}} & \mathbf{d}_{\gamma_{2}+1} & \cdots & \mathbf{d}_{n_{2}} & \cdots & \mathbf{b}_{\gamma_{1}} & & \mathbf{b}_{\gamma_{2}+1} \\
\mathbf{d}_{\gamma_{2}} & \cdots & \mathbf{d}_{n_{2}}
\end{array}\right),
\end{aligned}
$$

where

$$
\mathbf{c}_{k}= \begin{cases}\mathbf{e}_{2} & \text { if } k \in\left[\gamma_{2}\right] \\ \mathbf{e}_{1} & \text { otherwise }\end{cases}
$$

Then $J_{\widetilde{\mathcal{D}}^{\prime}}=J_{\widetilde{\mathcal{D}}}$ because $\widetilde{\mathcal{D}}^{\prime}$ can be obtained by an elementary row operation on $\widetilde{\mathcal{D}}$. Let $\delta=(0, \ldots, 0,-1) \in \mathbb{Z}^{d_{1}+d_{2}+2}$. Since the usual inner product $\delta \cdot\left(\mathbf{b}_{j}, \mathbf{d}_{k}, \mathbf{c}_{k}\right)$ equals

$$
\begin{cases}-1 & \text { if } k \in\left[\gamma_{2}\right] \\ 0 & \text { otherwise, }\end{cases}
$$

and $\delta \cdot\left(\mathbf{b}_{j}, \mathbf{d}_{k}, \mathbf{e}_{1}\right)=0$, it follows that a subring $K[\widehat{Z}] / J_{\mathcal{D}}$ of $K[Z] / J_{\widetilde{\mathcal{D}}^{\prime}}$ is a combinatorial pure subring of $K[Z] / J_{\widetilde{\mathcal{D}}^{\prime}}$ (see [9]). Thus $J_{\mathcal{D}}=J_{\widetilde{\mathcal{D}}^{\prime}} \cap K[\widehat{Z}]$. In particular, $N$ is a generating set (resp. a Gröbner basis) for $J_{\mathcal{D}}$. Furthermore, by elementary row operations on $\mathcal{D}$, we can obtain the integer matrix arising from $S\left(M_{1}, M_{2}\right)$ with respect to the basepoint $c$. Therefore $N$ is a generating set (resp. a Gröbner basis) for $J_{S\left(M_{1}, M_{2}\right)}$.

Theorem 3.3. Classes $\mathcal{M}_{\mathcal{Q G}}$ and $\mathcal{M}_{\mathcal{Q}}$ are closed under series and parallel connections and 2-sums.

Proof. Let $M_{1}$ and $M_{2}$ be matroids with $E_{1} \cap E_{2}=\{c\}$. Let $S\left(M_{1}, M_{2}\right)$ (resp. $P\left(M_{1}, M_{2}\right)$ ) denote a series (resp. parallel) connection of $M_{1}$ and $M_{2}$ with respect to the basepoint $c$.

In the case of series and parallel connections, if $c$ is a loop or a coloop of $M_{1}$ and the theorem holds for $M_{1}$ and $M_{2}$, then it also holds for $S\left(M_{1}, M_{2}\right)$ and $P\left(M_{1}, M_{2}\right)$. Suppose that neither $M_{1}$ nor $M_{2}$ has $c$ as a loop or a coloop. Then by Lemma 2.2 
and Lemma 3.1, $\mathcal{M}_{\mathcal{Q G}}$ and $\mathcal{M}_{\mathcal{Q}}$ are closed under series connections. Also, $\mathcal{M}_{\mathcal{Q G}}$ and $\mathcal{M}_{\mathcal{Q}}$ are closed under parallel connections from Proposition 1.4, and $P\left(M_{1}, M_{2}\right)=$ $\left[S\left(M_{1}^{*}, M_{2}^{*}\right)\right]^{*}$ for any matroids $M_{1}$ and $M_{2}$ [12, Proposition 7.1.14].

In the case of the 2-sum, since $M_{1} \oplus_{2} M_{2}=S\left(M_{1}, M_{2}\right) / c, \mathcal{M}_{\mathcal{Q G}}$ and $\mathcal{M}_{\mathcal{Q}}$ are closed under 2-sums.

Using the above results, we have

Theorem 3.4. Let $M$ be a matroid. If $M$ has no minor isomorphic to any of $M\left(K_{4}\right), \mathcal{W}^{3}, P_{6}$, or $Q_{6}$, then the toric ideal $J_{M}$ has a quadratic Gröbner basis.

Theorem 3.4 immediately holds from the following result:

Theorem 3.5 ([4, Corollary 3.1]). A matroid $M$ is a minor of direct sums and 2-sums of uniform matroids if and only if $M$ has no minor isomorphic to any of $M\left(K_{4}\right), \mathcal{W}^{3}, P_{6}$, or $Q_{6}$.

Let $M$ be a matroid on $E$, and let

$$
\text { rk : } 2^{E} \rightarrow \mathbb{Z}_{\geq 0} \quad X \mapsto\left|B_{X}\right|,
$$

where $B_{X}$ is a basis for $M \backslash(E-X)$. A function rk is said to be the rank function of $M$. Let $\lambda_{M}(X)=\operatorname{rk}(X)+\operatorname{rk}(E-X)-\operatorname{rk}(M)$ for $X \subset E$. We call $\lambda_{M}$ the connectivity function of $M$. For $X \subset E$, if $\lambda_{M}(X)<k$, where $k$ is a positive integer, then both $X$ and $(X, E-X)$ are called $k$-separating. A $k$-separating pair $(X, E-X)$ for which $\min \{|X|,|E-X|\} \geq k$ is called a $k$-separation of $M$ with sides $X$ and $E-X$. For all $n \geq 2$, we say that $M$ is $n$-connected if, for any $k<n$, it has no $k$-separation.

Any matroid which is not 3-connected can be constructed from 3-connected proper minors of itself by a sequence of the operations of direct sums and 2-sums. Therefore, in order to prove Conjecture 1.3, it is enough to prove the following conjecture:

Conjecture 3.6. For any 3-connected matroid $M$,

(1) $J_{M}$ is generated by quadratic binomial;

(2) $J_{M}$ has a quadratic Gröbner basis.

\section{ACKNOWLEDGEMENT}

The author would like to thank Hidefumi Ohsugi for useful comments and suggestions.

\section{REFERENCES}

[1] J. Blasiak, The toric ideal of a graphic matroid is generated by quadrics, Combinatorica, 28(3), (2008), 283-297.

[2] S. Blum, Base-sortable matroids and Koszulness of semigroup rings, Europ. J. Combin. 22 (2001), 937-951.

[3] J. Bonin, Basis-exchange properties of sparse paving matroids, Adv. in Appl. Math., 50 (2013), 6-15.

[4] B. Chaourar, "A characterization of uniform matroids," ISRN Algebra, vol. 2011, Article ID 208478, 4 pages, 2011. doi:10.5402/2011/208478. 
[5] A. Conca, Linear spaces, transversal polymatroids and ASL domain, J. Algebraic Comb., 25 (2007), no. 1, 25-41.

[6] A. Engström, T. Kahle and S. Sullivant, Multigraded commutative algebra of graph decompositions, J. Algebraic Combin., 39 (2014), no. 2, 335-372.

[7] K. Kashiwabara, The toric ideal of a matroid of rank 3 is generated by quadrics, Electron. $J$. Combin., 17, (2010), \#R28.

[8] M. Lasoń and M. Michałek, On the toric ideal of a matroid, Adv. Math., 259 (2014), 1-12.

[9] H. Ohsugi, A geometric definition of combinatorial pure subrings and Gröbner bases of toric ideals of positive roots, Comment. Math. Univ. St. Pauli, 56 (2007), no. 1, 27-44.

[10] H. Ohsugi, J. Herzog and T, Hibi, Combinatorial pure subrings, Osaka J. Math. 37 (2000), no. $3,745-757$.

[11] H. Ohsugi and T. Hibi, Compressed polytopes, initial ideals and complete multipartite grahs, Illinois J. Math. 44 (2000), no. 2, 141-146.

[12] J. Oxley, "Matroid theory," Second Edition, Oxford University Press, New York, (2011).

[13] J. Schweig, Toric ideals of lattice path matroids and polymatroids, J. Pure Appl. Algebra, 215 (2011), no. 11, 2660-2665.

[14] B. Sturmfels, "Gröbner bases and convex polytopes," Amer. Math. Soc., Providence, RI, 1996.

[15] B. Sturmfels, Equations defining toric varieties, Proc. Sympos. Pure Math. 62 (1997), 437-449.

[16] S. Sullivant, Toric fiber products, J. Algebra 316 (2007), no. 2, 560-577.

[17] N. White, The basis monomial ring of a matroid, Adv. Math. 24 (1977), 292-297.

[18] N. White, A unique exchange property for bases, Linear Algebra Appl. 31 (1980), 81-91.

Kazuki Shibata, Department of Mathematics, College of Science, Rikkyo UniVERSITY, TOSHIMA-KU, TOKYO 171-8501, JAPAN.

E-mail address: k-shibata@rikkyo.ac.jp 